$\mathrm{cm} \cdot \mathrm{min}^{-1}$ ) while attached to an artificial neck (a plastic beaker $10 \mathrm{~cm}$ in diameter with a $2 \mathrm{~cm}$ diameter tracheostomy). The neck strap was adjusted to a length of approximately $25 \mathrm{~cm}$ which resulted in a moderate tension, similar to that which might be adjusted clinically. The extubation force at which slippage occurred was recorded as the lock nut was adjusted by a varying number of turns. Two new samples (size \#8) and the one involved in the clinical report (size \#7) were tested in this way.

Slippage was observed at a force level of approximately $1 \mathrm{kgf}$ when the lock nut was adjusted by $3 \frac{1}{4}$ to $3 \frac{1}{2}$ turns for the clinical sample and one of the new samples. Although the torque required to tighten the locking nut was not quantified in these simulations, adjusting the locking screw by more than three turns required the flange to be held firmly against the rigid artificial neck. The torque required to tighten the nut became noticeable at about two turns with this new sample, while tightening by more than 3.5 turns required considerable effort. The other new sample required only about $2 \frac{1}{4}$ turns to achieve slippage at these force levels, but the torque was notably higher for the same number of turns.

Most clinical applications are unlikely to exceed the maximum torque levels used in the above simulations (i.e. $3 \frac{1}{2}$ turns), which means that unintentional traction of about $1 \mathrm{kgf}$ can result in the flange slipping with these products. Since breathing circuit disconnects occur at forces greater than $1 \mathrm{kgf}$, clinical incidents involving unintentional traction would be expected to exceed the threshold for slippage. We agree with the clinical report that the design needs to be modified before being considered acceptable for clinical use.

The Canadian distributor promptly issued a recall notice on this product when these concerns were brought to his attention.

\author{
Alex Sinclair PHD \\ Jos Van Bergen \\ Research and Standards Division \\ Bureau of Radiation and Medical Devices \\ Health Protection Branch \\ Health and Welfare Canada \\ Ottawa
}

\section{Determinants of FRC}

To the Editor:

The recent review by Wahba concerning the perioperative functional residual capacity (FRC) ${ }^{1}$ is in keeping with the many excellent educational articles published in this journal. However, I feel a number of statements made by Dr. Wahba require clarification. The first is the statement that partial curarization decreases FRC in supine subjects. Our observations ${ }^{2}$ as well as those of Holle et al. ${ }^{3}$ demonstrated that supine FRC is unchanged by the induction of rather marked degrees of weakness. The study of DeTroyer et al. ${ }^{4}$ cited in the review was performed in seated subjects. In this position the role of tonic inspiratory muscle tone is far more important in maintaining FRC than in the supine position.

The review also cites gas trapping behind closed airways as a major mechanism in the reduction of FRC with general anaesthesia. Rehder et al. ${ }^{5}$ discount this mechanism for FRC reduction since FRC measurements with $\mathrm{N}_{2}$ washout, which measure only gas in contact with open airways, and body plethysmography, which measures the entire volume of thoracic gas including that "trapped," indicated changes of similar magnitude. ${ }^{6}$

The importance of gas trapping is also alluded to in discussion of the effects of tracheal intubation of FRC. Indeed vigorous coughing may be associated with a reduction in FRC following tracheal intubation if gas dilution techniques are utilized. ${ }^{7}$ On the other hand, in patients with moderate airway obstruction, tracheal intubation was accompanied by a significant $(30 \%)$ increase in FRC as measured with helium dilution. ${ }^{8}$ These patients appeared to develop reflex airway constriction but there was no mention of coughing. Tracheal intubation in normal volunteers did not produce significant changes in FRC $^{9,10}$ despite evidence of reflex constriction in airways distal to the tube as suggested by airway resistance measurements. Therefore, there appears to be little reason to speculate that tracheal intubation may reduce FRC either by interfering with glottic action or by causing reflex airway constriction.

Thomas J. Gal MD

Department of Anesthesiology

University of Virginia Health Sciences Center

Charlottesville, VA 22908

\section{REFERENCES}

1 Wahba RWM. Perioperative functional residual capacity. Can J Anaesth 1991; 38: 384-400.

2 Gal TJ, Arora NS. Respiratory mechanics in supine subjects during partial curarization. J Appl Physiol 1982; 53: 57-63.

3 Holle RHO, Schoene RB, Pavlin EG. Effects of respiratory muscle weakness on $\mathrm{P}_{0.1}$ induced by partial curarization. J Appl Physiol 1984; 57: 1150-7.

4 DeTroyer A, Basternier J, Delhez L. Function of respiratory muscles during partial curarization in humans. J Appl Physiol 1980; 47: 1049-56.

5 Rehder K, Cameron PD, Krayer $S$. New dimensions of the respiratory system. Anesthesiology 1985; 62: 230-3.

6 Westbrook PR, Stubbs SE, Sessler AD, Hyatt RE. Effects of anesthesia and muscle paralysis on respiratory mechanics in normal man. J Appl Physiol 1973; 34: 81-6.

7 Bickler PE, Dueck R, Prutow RJ. Effects of barbiturate anesthesia on functional residual capacity and rib cage/diaphragm contributions to ventilation. Anesthesiology $1987 ; 66: 147-52$.

8 Matushima $Y$, Jones $R L$, King EG, Moysa $G$, Alton $J D M$. Alterations in pulmonary mechanics and gas 
exchange during routine fiberoptic bronchoscopy. Chest 1984; 86: 184-8.

9 Gal TJ, Suratt PM. Resistance to breathing in healthy subjects after endotracheal intubation under topical anesthesia. Anesth Analg 1980; 59: 27-4.

10 Rodenstein DO, Stanescu DC, Francis $C$. Demonstration of failure of body plethysmography in airway obstruction. J Appl Physiol 1982; 52: 949-54.

\section{REPLY}

Dr. T. Gal, who is well versed in respiratory muscle and small airway physiology, raises very specific points concerning the mechanism of reduced FRC during anaesthesia. Before answering, two considerations must be clarified:

- In a review of the literature designed for clinicians, as opposed to respiratory physiologists, the author often has to make hard decisions about what to include or exclude and how to present these data in the general context. Thus, some readers may disagree.

- Secondly, there are many instances, as will be seen below, when similar studies have yielded very different results. The difficulty then is to reconcile these different results.

The first point raised concerns the effects of d-tubocurarine on FRC. DeTroyer's 's work was quoted only to explain the role of inspiratory muscle tone in determining FRC and not to discuss the effects of curarization as such on FRC. It is true that curarization reduces $F R C$ only if the subject is seated. I regret this misunderstanding. The reader is referred to a lucid editorial which reconciled the differences between DeTroyer's data and that which Dr. Gal quotes above. ${ }^{2}$ The reconciliation allows the conclusion that the change in FRC is primarily due to loss of inspiratory muscle tone.

Next is the question of gas trapping. Rehder et al. reported a clear-cut relationship between closing volume and the degree of reduced FRC ${ }^{3}$ Later work, however, did not confirm that: an example of differing results we sometimes read.

I have looked very carefully at the data reported by the Mayo Clinic group ${ }^{4}$ and found no correlation between either the magnitude of change or the intraoperative FRC and closing volume. For that reason I was very careful in stating that gas trapping may be an additional contributing factor. Another example of the difficulties encountered is in reconciling Hickey's data (FRC is reduced more in patients with COPD) and Gunnarsson' ${ }^{6}$ work (no dimensional changes in CT scan in similar patients). The difference is, logically, due to trapped gas. It is in accepting this explanation that the bits of information can be placed in the general framework. I see Dr. Gal's points and I can but reiterate that gas trapping can only be an additional mechanism.

Richard W.M. Wahba MB BCH SCFRCPC Montréal, Que.

\section{REFERENCES}

1 DeTroyer A, Bastenier J, Delhez L. Function of respiratory muscles during partial curarization in humans. J Appl Physiol 1980; 49: 1049-56.

2 DeTroyer A, Martin JG. Respiratory muscle tone and the control of functional residual capacity. Chest 1983; 84: 3-4.
3 Rehder K, Mallow JE, Fibush EE, Krabill DR, Sessler $A D$. Effects of isoflurane anesthesia and muscle paralysis on respiratory mechanics in normal man. Anesthesiology $1974 ; 44: 477-85$

4 Juno $P$, Marsh $M$, Knopp TJ, Rehder $K$. Closing capacity in awake and anesthetized-paralyzed man. J Appl Physiol 1978; 44: 238-44.

5 Hickey RF, Visick WD, Fairley $H B$, Fourcade $H E$. Effects of halothane anesthesia on functional residual capacity and alveolar-arterial oxygen tension difference. Anesthesiology 1973; 38: 20-4.

6 Gunnarsson L, Tokics L, Lundquist $H$ et al. Chronic obstructive pulmonary disease and anaesthesia - formation of atelectasis and gas exchange impairment. Acta Anaesthesiol Scan (In Press).

\section{Uterine relaxation can be life saving}

To the Editor:

Uterine relaxation may be required in complicated obstetrical situations. Classically amyl nitrite, diethyl ether and halogenated hydrocarbons have been used. Recently, betamimetic drugs are being used. ${ }^{1-3}$ There are some reports of manual extraction of retained placentae using intravenous nitroglycerin to relax the uterus, ${ }^{4,5}$ but none, to our knowledge, of its use at Caesarean delivery to induce rapid uterine relaxation as a life-saving manoeuvre.

The patient presented at term, with a double footling breech in active labour. A Caesarean section was performed under epidural anaesthesia. At delivery, the baby's head was trapped in the hypertonic upper segment. While we did not document the duration of these events it became obvious that the baby was in jeopardy. The anaesthetist gave $1000 \mu \mathrm{g}(1 \mathrm{mg})$ of nitroglycerin intravenously. It was only then that the uterus became sufficiently relaxed to allow delivery of the head. The patient remained asymptomatic although her blood pressure did decrease to $70 / 30$ but this responded to $36 \mathrm{mg}$ of ephedrine in divided doses.

The Apgar scores were five and nine at one and five minutes respectively. The infant breathed room air by two minutes of age and weighed $3090 \mathrm{~g}$. Cord blood gas analysis was not measured.

This patient's obstetrical problem was life-threatening and its management unique. Rapid uterine relaxation was essential. All the methods used to induce uterine relaxation have potential problems - e.g., delay in onset of action, increased bleeding, risks of emergency anaesthetic induction and subsequent deep general anaesthesia.

It is our impression that intravenous nitroglycerin relaxes the uterus quickly, effectively and safely. Further 